\title{
Three Dimensional Chemical Mapping of Fly Ash
}

\author{
Quinang $\mathrm{Hu}^{\mathrm{a}}$, Robert Frazier ${ }^{\mathrm{a}}$, Tyler Ley ${ }^{\mathrm{a}}$, Jay Hanan ${ }^{\mathrm{a}}$, Jeff Davis ${ }^{\mathrm{b}}$ \\ ${ }^{a}$ Oklahoma State University \\ ${ }^{\mathrm{b}}$ National Institute of Standards and Technology
}

Over 6 billion cubic yards of concrete are placed annually throughout the world. The widespread use of concrete is attributed to its low cost, wide availability, flexible form, and its long term durability. One of the primary materials used to improve the durability, economy, sustainability, and constructability of concrete is fly ash. Fly ash is a waste product from coal fired power plants. Typically, fly ash particles are spherical and between $1 \mathrm{~mm}$ to $1 \mathrm{~mm}$ in diameter. Fly ash consists of reactive amorphous phases and inert crystalline phases that are mixed within the particle. Currently, bulk methods of XRF analysis are used to determine the chemical makeup of fly ash. While these bulk methods of analysis are useful, the authors feel that more information can be learned if the phases within a fly ash particle could be mapped and their distribution within the particle better understood. It would be expected that phases near the perimeter of the particle would play a more significant role in determining the performance of the fly ash as it will be the first to react.

There are very few efficient and reliable methods for creating 3D compositional images of particles. In this presentation, the authors suggest a method that combines two imaging techniques. In the first technique a 3D map of a fly ash particle is created by using X-ray micro Computed Tomography (mCT) represented slice from the mCT investigation is shown in Figure 1 on the left. Next the particle is sectioned and polished down to a random chord. This exposed surface is then analyzed with an electron microprobe with energy dispersive spectrometry (EM/EDS) for the elements present. A full spectrum was acquired at each pixel in the image, and the hyperspectral datacube was interrogated to derive phase maps for each particle using simple threshold based classification. Next the mCT data was interrogated to find the same cross-section as was investigated in the EM/EDS analysis. By overlaying these images one is able to find the corresponding gray values in the mCT analysis that match with the phases identified with the EM/EDS analysis. By doing this it allows the extrapolation of the 2D data from the EM/EDS to the 3D model of the fly ash particle produced with the mCT. This model is shown in Figure 2.

Finally a novel dissolution algorithm is used to look at the distribution of the phases within the fly ash particle with depth away from the surface based on the segmented 3D model. A result from this analysis is shown in Fig. 3.

An overview of this process will be presented along with a summary of the available data. Future applications of this technique will also be presented. 

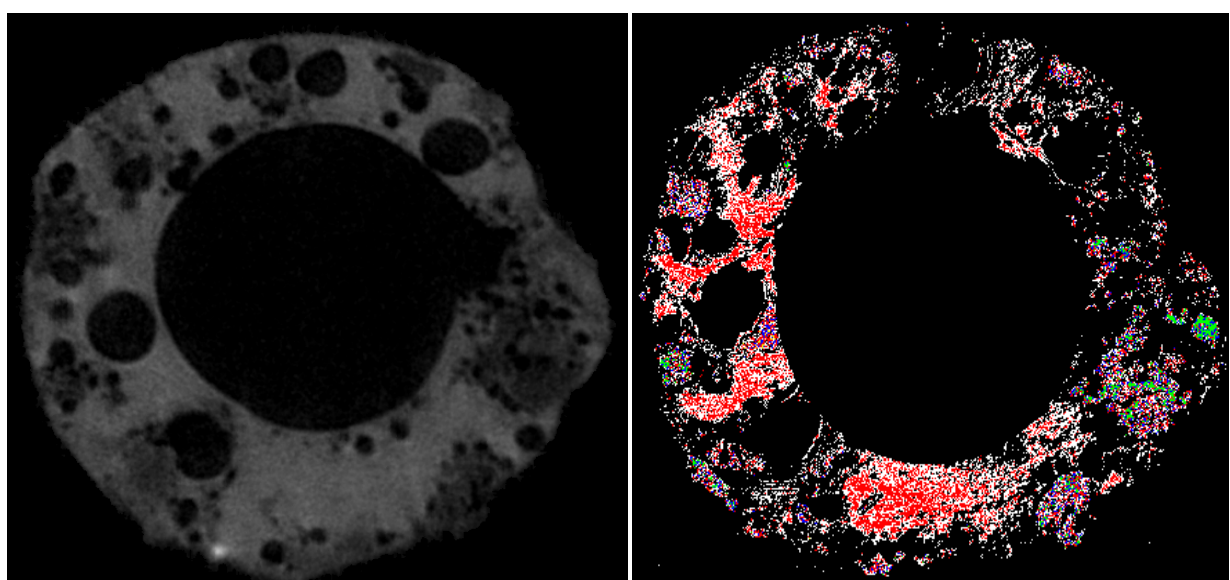

Figure 1 - The segmented image from the EM/EDS chemical analysis is shown on the right with the corresponding X-section from the tomography data on the left.

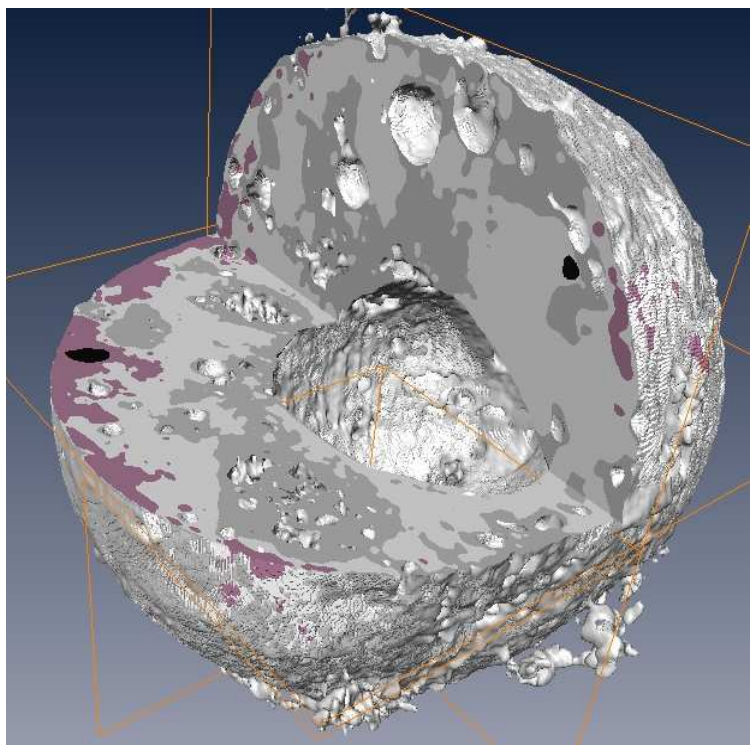

Figure 2 - A segmented image of a fly ash particle showing the location of different phases.

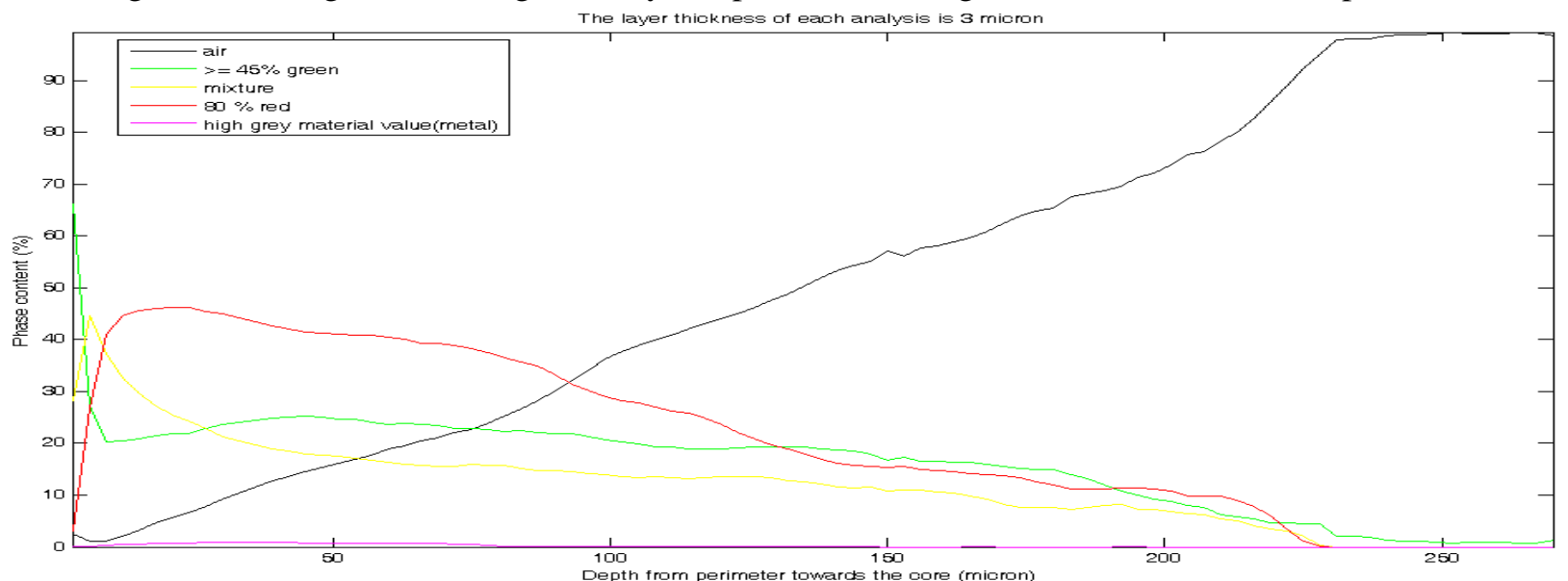

Figure 3 - An analysis of the chemical phases with depth of the fly ash particle. 